\title{
Identifying the Muscle Synergy Pattern during Human Grasping
}

\author{
${ }^{1}$ Afsaneh Koohestani, ${ }^{2}$ Hamid Reza Kobravi and ${ }^{3}$ Mahereh koohestani \\ Islamic Azad University of Mashhad, Iran \& the Institute of training, research, healthcare of Ghaem, Iran \\ ${ }^{1}$ afsaneh_koohestani@ymail.com; ${ }^{2 h}$ kobravi@gmail.com; ${ }^{3}$ mahereh.koohestani@gmail.com
}

\begin{abstract}
In this work, a methodology has been proposed and evaluated for identification the muscle synergy patterns during human grasping. The proposed approach is based on decomposition analysis of involved muscle activation profiles utilizing the Hierarchical Alternating Least Squares (HALS) algorithm. The surface EMG signals of Flexor Digital Superfacialis and Flexor Pollicis Longus muscles were recorded during grasping a cylindrical object. EMG signals were full-wave rectified and smoothed through a low pass filter. Then the HALS algorithm was utilized for decomposition of muscle activation profiles. The HALS algorithm can be efficiently used instead of NNMF (non-negative matrix factorization) method. The HALS method not only provides a very good convergence property but also there is not the nonnegativity constraint for the decomposed factors. The results of evaluations are interesting and promising.
\end{abstract}

Key words: Surface EMG, Hals algorithm, muscle synergy

\section{Introduction}

A functional muscle synergy means the pattern of co-activation of muscles recruited by a single neural command signal [2]. The central nervous system (CNS) uses multiple joints and muscles to perform the stable and flexible goal-directed movements. Existence of muscle synergies as a neural strategy has been proposed to justify how the CNS can solve the problem of redundancy and how it copes with complexity of control problem [1,7and13].

In recent years, many evidences have been presented proving modularity during the performance of different motor tasks in humans and other animals [3, 4]. According to modular control hypotheses, the motor primitives that are organized at the spinal level are modules that in a linear or non-linear manner can be combined to produce enormous collection of movements [2]. Muscle synergies are the possible candidates for motor primitives [10]. It is also has been shown that the decomposition of EMG envelope can be resulted in identifying a small number of muscle synergies $[7,14]$. There are two main models for muscle synergies called synchronous muscle synergies and asynchronous muscle synergies [7, 8, and 9].

Recently, Castellini et al. [6], have presented the evidences of synchronous muscle synergies during human grasping using Principal Component Analysis (PCA). In this paper, we focused on human hand grasping while the wrist joint is at rest position. We analyzed the recorded SEMG signals of two muscles being involved in grasping a cylindrical object. We used NNMF method for EMG decomposition. Because it does not impose orthogonally and statistical independence (such as PCA and ICA respectively) $[8,9]$ 
and it seems more suitable for extracting the entire muscle synergies[5]. Nevertheless, the standard NNMF applies non-negativity constraint for the decomposed factors. Since, smoothed full-rectified EMG are used for decomposition, the modified EMG may has the negative values for no ideality of smoothing filters. So, in this paper, the HALS algorithm has been used instead of NNMF method [12]. The HALS method dose not applies such constraint. In addition, using The HALS results in achievement both better accuracy and repeatability [11].

The archived results show the acceptable performance of proposed methodology.

\section{Method and Materials}

\subsection{Muscle synergy model}

In the synchronous synergy model, the EMG can be decomposed as a linear combination of set of vectors capturing a specific balance of muscle activations [7], by a time-variant activation coefficient.

$$
M(t)=\sum_{i=1}^{k} c_{i}{\overrightarrow{(t) \cdot w_{i}}}
$$

$M$ Represent the time-varying muscle activation pattern of $d$ involved muscles. $\overrightarrow{w_{l}}$ is the $i$ th $d$ dimensional basis vector. $c_{i} \overrightarrow{(t)}_{i}$, is the scalar activation coefficient for the $i$ th basis vector.

In fact, each $\vec{w}_{i}$ represents a muscle synergy related to the synaptic weights from premotor neurons to different motor neuronal pools, each $c_{i}$ represents the synergy activation coefficient or firing frequency recruiting a synergy.

\subsubsection{Non-Negative Matrix Factorization}

Most non-negative matrix decomposition methods are attributed to researchers such as Lee a Seung in which is the observation matrix [13]. Let's suppose:

$$
\begin{gathered}
M=C_{ \pm} W_{ \pm}+E \\
W=[w 1, w 2, \ldots w j] \in R_{+}^{I \times T} \\
C=[c 1, c 2, \ldots, c j] \in R_{+}^{I \times T}
\end{gathered}
$$

Where $\mathrm{W}$ and $\mathrm{C}$ are matrices containing the basis vectors and activation coefficients and $\mathrm{E}$ represents estimation error.

Let's define a cost function for $a w_{j}$ and its corresponding coefficient $c_{j}$ using the Frobenius norm as bellows:

$$
D_{F}^{j}\left(M^{j} \| c_{j} w_{j}\right)=\frac{1}{2}\left\|M^{j}-c_{j} w_{j}\right\|_{F}^{2}
$$

\subsection{HALS Algorithm}

In this manner we will have a bank of local cost functions. By minimizing the cost functions consecutively, based on the optimality conditions (KTT) [12], the stationary points of local cost function can be estimated using the gradient of local cost functions as follows: 


$$
\begin{gathered}
\nabla_{C_{j}} D_{F}^{j}\left(M^{(j)} \| c_{j} w_{j}^{T}\right) \geq 0 \\
\nabla_{w_{j}} D_{F}^{j}\left(M^{(j)} \| c_{j} w_{j}^{T}\right) \geq 0
\end{gathered}
$$

Where the gradient of the local cost function can be estimated as follows:

$$
\begin{aligned}
& \nabla_{c_{j}} D_{F}^{j}\left(M^{j} \| c_{j} w_{j}\right)=\frac{\partial D_{F}^{j}\left(M^{j} \| c_{j} w_{j}\right)}{\partial c_{j}}=c_{j} w_{j}^{T} c_{j}-M^{j} w_{j} \\
& \nabla_{w_{j}} D_{F}^{j}\left(M^{j} \| c_{j} w_{j}\right)=\frac{\partial D_{F}^{j}\left(M^{j} \| c_{j} w_{j}\right)}{\partial w_{j}}=c_{j}^{T} c_{j} w_{j}-M^{j} c_{j}
\end{aligned}
$$

If it is assumed the cj and wj are positive number then the stationary points can be estimated using the update laws as follows:

$$
\begin{aligned}
& w_{j} \leftarrow \frac{1}{c_{j}^{T} c_{j}}\left[M^{j^{T}} a_{j}\right]_{+}=\frac{1}{c_{j}^{T} c_{j}} \max \left\{\varepsilon, M^{j} w_{j}\right\} \\
& w_{j} \leftarrow \frac{1}{c_{j}^{T} c_{j}}\left[M^{j^{T}} a_{j}\right]_{+}=\frac{1}{c_{j}^{T} c_{j}} \max \left\{\varepsilon, M^{j} w_{j}\right\}
\end{aligned}
$$

Where $\varepsilon$ is a small positive number, above update rules are known as the HALS algorithm was proposed by Chikoki et al. [14].

\subsection{Data Collection and Preprocessing}

Ten healthy participants, men and women, are asked to hold a one-kilogram cylinder in their hands for 10 seconds and then drop it, and repeated it again for five times. During the experiments the wrist joint of the participants were at the rest posture. The recorded EMG signals was rectified and passed through a low-pass filter with $20 \mathrm{~Hz}$ cut-off frequency. Then, the obtained signals were normalized.

\section{Results}

One of most prevalent methods being used to determine the number of synergies is based on R2 criterion. R2 represents the fraction of total variation accounted for by the synergy reconstruction (Eq. 12), [15].

$$
R^{2}=1-\frac{S S E}{S S T}=1-\frac{\sum_{s} \sum_{k=1}^{k_{j}}\left\|m^{j}\left(t_{k}\right)-\sum_{i} c_{i}^{j} w_{i}\left(t_{k}-t_{i}^{j}\right)\right\|^{2}}{\sum_{s} \sum_{k=1}^{k_{j}}\left\|m^{s}\left(t_{k}\right)-\bar{m}\right\|^{2}}
$$

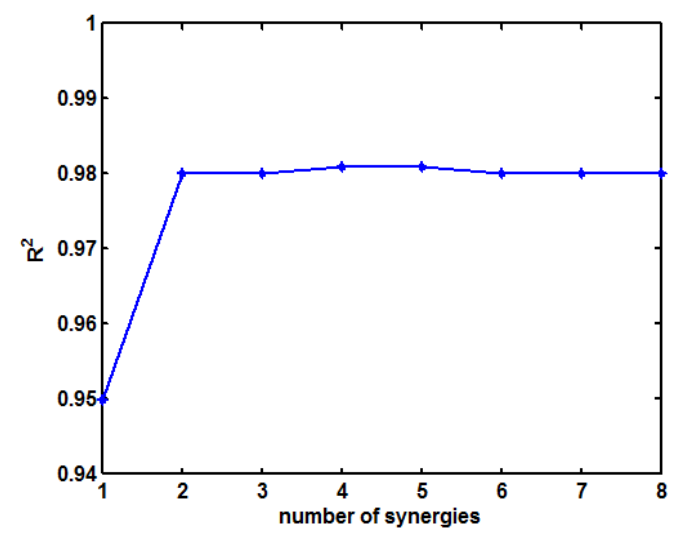

Figure 1: R2 changes versus the number of synergies. 
Where SSE is the sum of the squared residuals, SST is the sum of the squared residual from the mean activation vector $(\bar{m})$. As the Fig.1 indicates, the slope of $\mathbf{R}^{2}$ changes has been decreased considerably at 2. So, in this work the number of selected synergies is 2 .

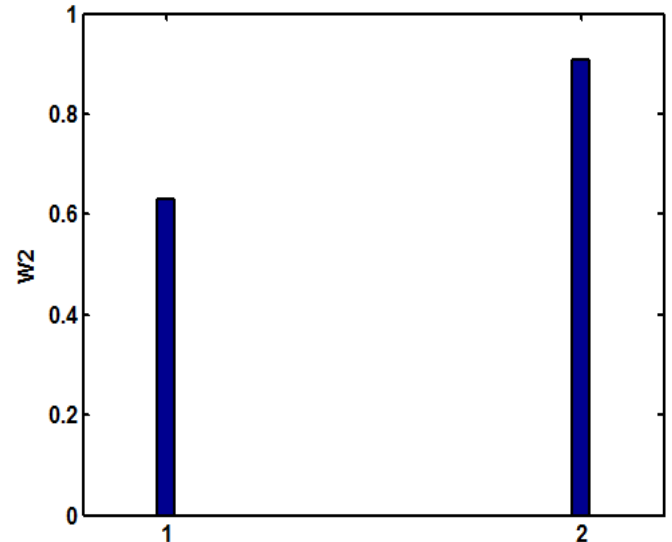

(A)

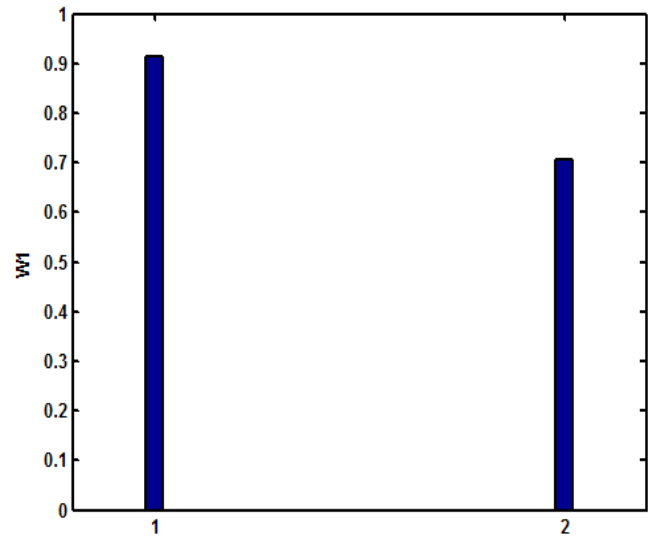

(B)

Figure 2: Shows the activation level of the two involved muscles corresponding to each synergy pattern

In the next step, basis vectors and activation coefficients were estimated using HALS algorithm described in the previous section. Figure 2, shows the activation level of the two involved muscles corresponding to each synergy pattern.

\section{Discussion}

In figure 3, the muscle synergy pattern during object grasping was extracted using analyses of computed muscle activation profiles relating to two involved muscles. In this research, HALS algorithm and NNMF were utilized for identifying the synergy vectors.

HALS algorithm is known as a fast convergent approach without non-negativity constraint for the decomposed factors $[11,14]$. In the other hand, the main purpose of the present study was to achieve the proper muscle synergy patterns by solving the problem of nonnegative matrix analysis. For quantitative evaluation of the propose approach, the root mean square (RMS) of the error signal, the difference between the reconstructed muscle activation profiles and actual activation profiles, were calculated [Table 1]. The calculations show the low difference between the actual and reconstructed signals. This can be due to the efficiency of HALS algorithm; however there is difference between actual signal and reconstructed signal by NNMF algorithm. 

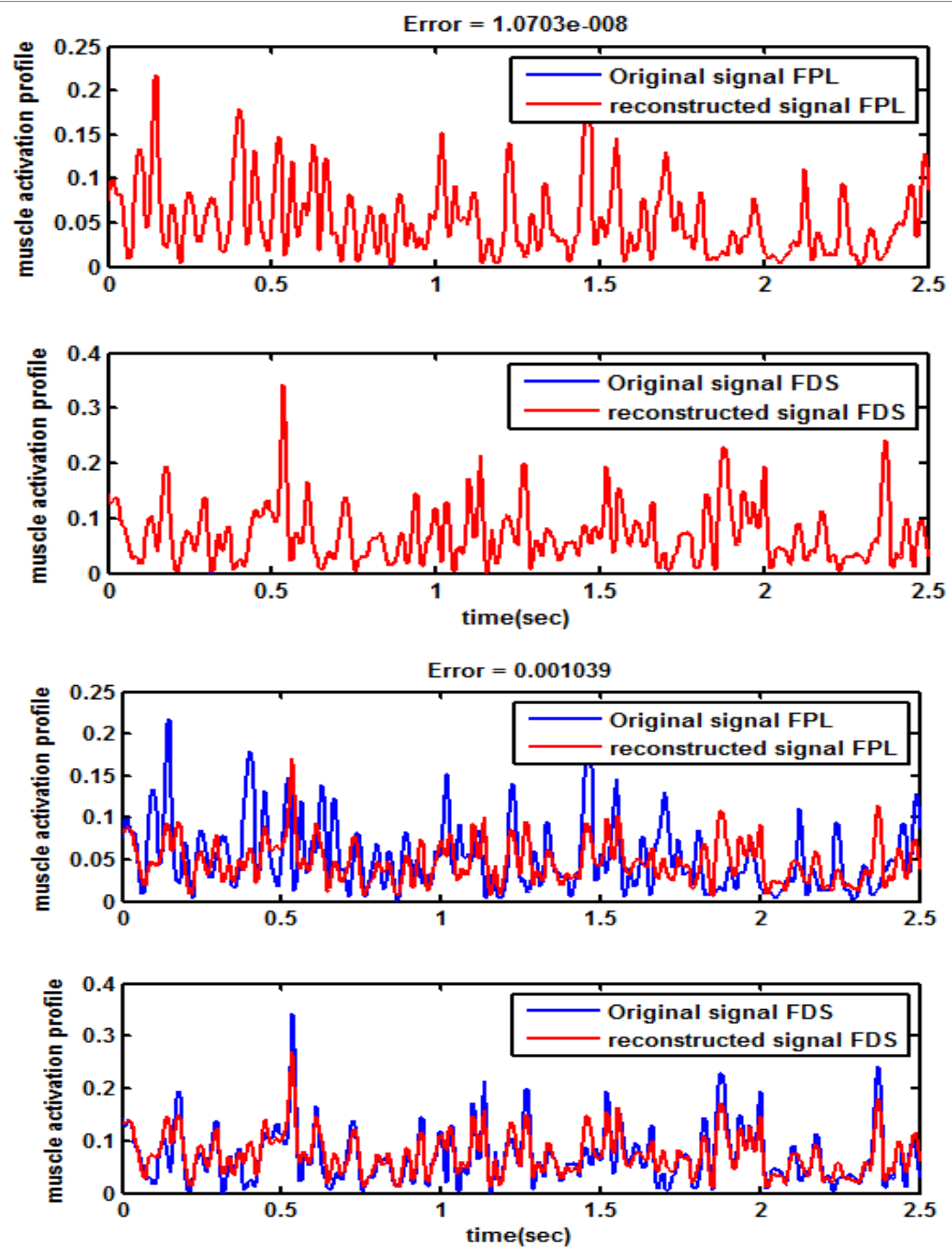

Figure 3: Comparing the reconstructed muscle activation with the original signals by 3 numbers of synergy, (A) demonstrate HALS algorithm and (B) demonstrate NNMF. FDS: demonstrates signals of Flexor Digital Superfacialis, FPL: demonstrates signals of Flexor Pollicis Longus

Table 1: Demonstrate RMS of both algorithm (Hals, NNMF) for Flexor Digital Superfacialis, Flexor Pollicis Longus

\begin{tabular}{|ccccc|} 
Subject & $\begin{array}{c}\text { Rms(Hals) } \\
\text { FDS } \times \mathbf{1 0}^{-8}\end{array}$ & $\begin{array}{c}\text { Rms(Hals) } \\
\text { FPL } \times 1^{-8}\end{array}$ & $\begin{array}{c}\text { Rms(NNMF) } \\
\text { FDS } \times 1^{-4}\end{array}$ & $\begin{array}{c}\text { Rms(NNMF) } \\
\text { FPL } \times 1^{-4}\end{array}$ \\
\hline Male1 & 1.1511 & 1.2334 & 2.3647 & 2.2103 \\
Male2 & 1.4567 & 1.9712 & 2.1999 & 2.3614 \\
Male3 & 1.8835 & 1.3451 & 3.8041 & 3.1200 \\
Male4 & 1.3260 & 1.0241 & 2.3330 & 2.3212 \\
Male5 & 1.1249 & 1.7503 & 3.0100 & 3.0144 \\
Female1 & 1.0241 & 1.0021 & 2.4851 & 2.4851 \\
Female2 & 1.7503 & 2.0144 & 3.3541 & 3.3541 \\
Female3 & 1.0021 & 1.0222 & 3.0144 & 1.0039 \\
Female4 & 2.0006 & 2.0314 & 1.4729 & 3.1533 \\
Female5 & 1.0703 & 1.3766 & 2.0342 & 2.0342 \\
\hline
\end{tabular}



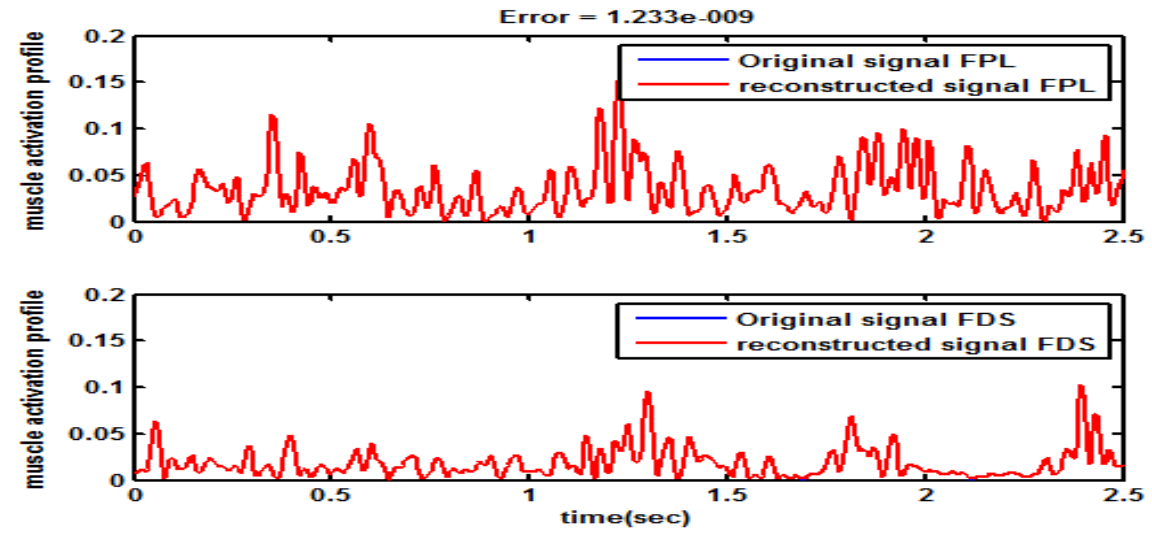

Figure 4: Comparing the reconstructed muscle activation with the original signals by 2 numbers of synergy, HALS algorithm

If number of synergies over estimated, we are going to compare the performance of reconstruction method base on HALS algorithm.

In figure.4, showed that when the number of synergies are over estimated (more than 2 synergies), there was not seen any difference between the actual signal and reconstructed signal. After identifying the synergy vectors, the activation profile of two involved muscles were reconstructed using the estimated basis vectors and coefficients. The reconstructed profiles were compared to the actual activation profiles. Figure 3, 4 and table 1, show reconstructed muscle activation profiles, computed, accompanied by actual computed muscle activation profiles. This research illustrated for the first time, it could be reconstructed signals by the minimizing of the number of synergies like 2 synergies.

Identifying the muscle synergy patterns, quantifying the modular organization of motor control, can provide insights on novel diagnostic and rehabilitation tools [11]. The achieved results prove the presented method has potential application to the neuron- rehabilitation.

\section{Result}

Difference between the reconstructed muscle activation profiles and actual activation profiles, were calculated for ten participants in hand grasping. The calculations showed the low difference between the actual and reconstructed signals. This can be due to the efficiency of HALS algorithm.

\section{ACKNOWLEDGMENT}

This work was supported by Neuromuscular Control Lab, biomedical department, Islamic Azad University, Mashhad branch, and also supported by physiotherapy department, Ghaem hospital, Mashhad University of Medical Sciences.

\section{REFERENCES}

[1] M. C. Tresch and A. Jarc,. "The case for and against muscle synergies". Current Opinion in Neurobiology".19, 601-607, 2009. 
Afsaneh Koohestani, Hamid Reza Kobravi, Mahereh koohestani; Identifying the Muscle Synergy Pattern during Human Grasping, Journal of Biomedical Engineering and Medical Imaging, Volume 1, No. 6, Dec (2014) , pp 33-39

[2] Bernstein, N. "The coordination and regulation of movements". Pergamon Press, Oxford.1967.

[3] Lacquaniti, F., Ivanenko, Y.P., Zago, M. "Patterned control of human locomotion". J. Physiol. 590, 2189-2199.2012.

[4] Bizzi, E., Cheung, V.C., d'Avella, A., Saltiel, P., Tresch, M. "Combining modules for movement". Brain Res. Rev. 57, 125-133. 2008.

[5] D. Ioannis, B. Bastien, P. Thierry and P. Stefano, "A methodology for assessing the effect of correlations among muscle synergy activations on task-discriminating information", Frontiers in Computational Neuroscience, 7, 2013, Article 54.

[6] Castellini, C., van der Smagt, P. "Preliminary evidence of dynamic muscular synergies in human grasping". In: Proceedings of ICAR -International Conference on Advanced Robotics, 28-33 (2011).

[7] A. d'Avella, P. Saltiel and E. Bizzi, "Combinations of muscle synergies in the construction of a natural motor behavior", Nat Neurosci, 6, 300-308. 2003.

[8] L. Omlor and M. A. Giese, "Anechoic blind source separation using wigner marginal" J. Mach. Learn.Res., 12, 2011, 1111-1148.2011

[9] A. d'Avella, L. Fernandez, A. Portone and F. Lacquaniti, "Modulation of phasic and tonic muscle synergies with reaching direction and speed", J. Neurophysiol, 100, 1433-1454, 2008.

[10] E. Bizzi, A. D’Vella, P. Saltiel and M. Tresch, "Modular organization of spinal motor systems", Neuroscientist, 8,437-442, 2002.

[11] A. H. Phan and A. Cichocki, "Multi-way nonnegative tensor factorization using fast hierarchical alternating least squares algorithm (HALS)", In Proc. of the 2008 International Symposium on Nonlinear Theory and its Applications, Budapest, Hungary, 2008.

[12] Cichocki, A., Zdunek, R., \& Amari, S. I., "Hierarchical ALS Algorithms for Nonnegative Matrix and 3D Tensor Factorization", in ICA07, London, UK, September 9-12, Lecture Notes in Computer Science, 2007,4666, pp. 169-176.2007.

[13] Lee, D. D., \& Seung, H. S., "Algorithms for Nonnegative Matrix Factorization", Advances in neural information processing systems, 13, 556-562,2001.

[14] A. Cichocki, R. Zdunek, A. H. Phan and S. Amari, "Nonnegative Matrix and Tensor Factorizations", John Wiley \& Sons Ltd: Chichester, UK, 2009.

[15] Andrea d'Avella, Alessandro Portone, Laure Fernandez and Francesco Lacquaniti, "Control of fast-reaching Movements by Muscle synergy combinations. J.Neuroscience, 26,77917810, 200 